\title{
Leveraging the Internet of Things for Energy Applications
}

\author{
Nikolay Kasatikov ${ }^{1, *}$, Oleg Brekhov ${ }^{1}$, Alexander Sytov $^{2}$, and Ekaterina Milovanova ${ }^{1}$ \\ ${ }^{1}$ MAI.Moscow Russia Volokolamskoe 14 \\ ${ }^{2}$ NIITP Moscow Russia Dekabristov 14
}

\begin{abstract}
The history of the development of such a thing as the Internet of things in our country, as well as in the world, is gaining momentum. The communication of all devices that maintain communication and transmit data online are used constantly and around the clock. In order to accumulate data and combine it for the benefit of local residents, as well as advertising companies, in order to understand their needs and solve with the help of unobtrusive offers, all data obtained from gadgets and devices connected to the Internet are used.
\end{abstract}

\section{Idea project}

\subsection{Creating the concept}

Prerequisites for creating the concept of a software system for processing and analyzing big data IoT devices for assessing the development of human settlements.

In recent years, there has been a rapid development of mobile sensor applications based on innovative technologies related to ubiquitous and pervasive computing, geographic information, crowdsourcing and social networks. We are currently living in the next digital generation of social media, where communication and interaction for user-generated content is primarily focused on improving the sustainability of smart cities. Thus, urban computing is defined as a technology for collecting, integrating, and analyzing large and heterogeneous data generated by various sources in urban spaces, such as sensors, devices, vehicles, buildings, and people, to solve the main problems that cities face. Moreover, this technology makes it possible to increase efficiency in responding to the needs of citizens.

The rapid growth of population density in urban cities requires the provision of new services and infrastructure to meet the needs of urban residents. Thus, there is an increasing demand for embedded devices such as sensors, actuators, smartphones, etc., which provides great business potential for the new era of the Internet of Things (IoT), in which all devices are able to connect and communicate with each other over the Internet. Thus, Internet technologies provide a way to integrate and share a common communication environment for joint urban systems [1].

The development of science and technology, the process of urbanization is accelerating all over the world, which, on the one hand, improves the quality of life of people, on the other hand, gives rise to serious problems such as pollution, traffic jams and constantly growing energy consumption. As data collection becomes easier and cheaper, a wider range of big data in urban space, such as data on human mobility and air quality, is being generated and made available.

This data allows us to solve the problems we face and help build smarter cities. For example, we can analyze city traffic jams based on GPS trajectories received from taxis, monitor and predict air quality based on the analysis of data received from air monitoring stations, traffic data, etc. The constructed models can be used when making decisions on the development of the road structure of cities. Thus, urban computing is becoming increasingly sought after by researchers in various problem areas related to the design and development of smart cities of the future.

The main methods and technologies of designing and developing software systems for urban computing, methods of fusion of knowledge from heterogeneous data sources and visualization of urban data are considered.

Purpose of the study. Development of theoretical research related to the methodology of design and development of software systems for processing and analyzing big data of IoT devices for assessing the development of settlements.

The revolution in mobile urban data monitoring is nearing completion, and we will soon see such software systems in everyday use.

\subsection{Function program}

How does the Internet of Things work?

The Internet of Things (IoT) connects devices into a computer network and allows them to collect, analyze, process and transmit data to other objects through software, applications or technical devices

IoT devices function on their own, although people can configure them or provide access to data. IoT systems operate in real time and usually consist of a

* Corresponding author: nick925@yandex.ru 
network of smart devices and a cloud platform to which they are connected via WiFi, Bluetooth, or other forms of communication.

Urban Computing is a new research area that combines research related to urban infrastructure management, transport logistics, civil engineering, environment, economics, ecology and sociology. This paper discusses the problems and issues related to the development and implementation of the concept of urban computing based on the analysis of big data from sensors of IoT devices.

The field combines information technology and analysis and data mining techniques with analysis and design techniques in areas such as civil engineering, transport logistics, economics, energy and the environment, which usually lead to complex analytical problems in real-world applications. Therefore, fully automatic analysis is difficult, often requiring considerable experience and in-depth knowledge in various fields. It is important to incorporate human perception into the data mining process and combine flexibility, creativity and knowledge of the human subject area with the enormous memory capacity and computing power of modern computers.

Such actions cannot be performed with ordinary people. They will send information at a convenient time and from a convenient place. There may also be a problem of lack of people, and therefore the necessary information, in the right place at the right time. This can lead to a missing or sparse data issue. Sometimes there can be a problem with an overabundance of data received from people in a certain place (a popular place with a large number of people), which in turn leads to an additional load on the systems for collecting, storing and processing data. If people partially transfer data from their mobile devices (geolocation is disabled, time stamping is not applied, etc.), then this can lead to incomplete data sampling from users. Consequently, there may be differences between the distribution of the full dataset and the distribution of partial data, this is greatly influenced by the movement of people.

The development of urbanization, the emergence of single-industry towns led to a rapid change in the lives of many people. But this progress also influenced the increase in old and the emergence of new problems associated with the functioning of cities. Such problems include an increase in traffic congestion, an increase in energy consumption, as well as environmental pollution, including a decrease in air quality, etc. These problems are associated with the complex dynamic processes of the functioning and development of cities, therefore, for decades, these problems were considered unsolvable.

A large amount of data is generated by various sources, such as a variety of sensors, mobile devices as well as IoT devices, vehicles. Thus, the collection, aggregation and analysis of this data in order to solve the main problems facing cities (for example, air pollution, increased energy consumption and traffic congestion), we will call urban computing.

\subsection{Creating program}

The idea of creating a software system for processing data from IoT devices

Facility security is key to the country's viability. The need to control these facilities forces us to look for new measures and new control parameters. Now the technology of the Internet of Things is developing, in our opinion it is ideal for monitoring the life support objects of the region.

The idea to use IoT technologies appeared after studying their popularity and in connection with their convenience in many modern processes. One of the main advantages of this system is that IoT devices work independently, despite the fact that the operator can configure them, provide access to data, and also make adjustments. IoT systems operate in combat time and often consist of a network of smart devices and a cloud platform. If we talk about how they will work in our system, then at first the devices will collect data on energy facilities, TV towers and others. Further, our software will process them.

It is necessary to take into account that for the correct operation of the entire project, good analytics is needed, which will include all the components of the objects under study and the influence of the environment, for example, by studying space images and other data of remote sensing of the earth (ERS). Also, the message will touch upon such concepts as BigData, Connection, Devices, Experience. The report will describe how these technologies are currently used in the power industry, and more specifically, how the Internet of Things improves the controllability of substations and power lines through remote monitoring.

In addition, areas where IoT technologies are already used, for example, medicine, will be analyzed. As well as security and protection against attacks by cybercriminals.

In addition to Big Data, analytics, connections, devices and experience are also important for the Internet of Things. For simplicity, this principle is presented as ABCDE: Analytics, BigData, Connection, Devices, Experience.

\subsection{Example}

Analytics (analytics) is a key link in the functioning of the IoT, which unites the devices themselves, data from them, and optimizes business processes;

BigData - that is, information from devices - is stored in the cloud. They allow you to automate existing processes or build new ones;

Connection - These are the channels through which devices receive and transmit information;

Devices - devices connected to the system, which for correct operation, depending on the tasks, must have an appropriate frequency of messages;

Experience (experience) - working with the existing experience of solving customer problems using the IoT, its analytics and rethinking.

\section{Big Data theory}


In the power industry, the Internet of Things improves the controllability of substations and transmission lines through remote monitoring.

\section{Procces example}

\subsection{Process examples}

Today, most mobile phones include various sensors such as GPS, accelerometers, microphones, and cameras. МоделиClassification models can use data from these sensors to evaluate user actions and environmental events. Such models can drive key areas of mobile applications, including mobile healthhealth and green energy awareness awareness, to name just a few. However, in the real world, there are significant problems associated with modeling human activity. For example, a key obstacle is differences in contextual conditions and user characteristics that occur in largescale mobile monitoring systems. This leads to distinctive features in the sensory data used by classifiers to recognize different types of human activity, varying from user to user. Today, the world is becoming increasingly urbanized, with about 50 percent of the world's population living on only a smaller percentage of the Earth's surface, perhaps just one percent. This urbanization is likely to grow by at least 70 percent in the coming years. Thus, cities have become one of the most complex artifacts. The rapid development of information technologies generates a huge amount of information that reflects various features of our life over the past decade. The digital footprints that come from our daily interactions with computing devices are valuable data sources for identifying urban issues.

This raw data can be used to develop data analysis tools to improve our understanding of urban dynamics and, consequently, improve the efficiency of urban systems. The growing use of mobile devices, including mobile phones and GPS navigation systems, is creating a new theory of monitoring, in which people serve as distributed sensors in the city, designed to measure urban dynamics. Consequently, smart cities are designed to improve public services.

\subsection{Area lot}

In healthcare, IoT allows you to move to a new level of diagnostics of diseases - "smart" devices monitor the patient's health indicators in the background.

In agriculture, smart farms and greenhouses meter fertilizer and water themselves, and smart animal trackers notify farmers in time not only about the location of animals, but also about their health status by analyzing heart rate, body temperature and general activity.

In transportation, typical IoT solutions include telematics and smart fleet management, where the vehicle connects to a local operating system for monitoring and diagnostics. According to Statista [2], \$ 740 billion will be invested in the development of IoT for cars by 2025 .
In urban environments, IoT solutions help automate lighting while reducing lighting costs by up to $30-50 \%$. Smart meters, which themselves record and transmit information on costs and wear and tear to management companies, relieve city dwellers from the need to verify and send data on energy consumption themselves.

In logistics, IoT reduces the cost of transportation and minimizes the impact of the human factor. IoT systems can also monitor garbage can fill and optimize waste collection costs based on this data.

The oil and gas and mining industries are actively adopting the Internet of Things. In particular, the use of in-depth analytics on boreholes is helping the oil and gas industry to increase production from already depleted fields. And, for example, Severstal, using the Internet of Things, was able to minimize the company's losses due to erroneous forecasts for electricity consumption.

In retail, IoT enables brands and sellers to optimize costs and improve customer experience through digital signage, customer interaction tracking, inventory management and smart vending machines, according to Maxim Lipatov. The market is expected to grow from \$ 14.5 billion in 2020 to $\$ 35.5$ billion by 2025 , according to Research And Markets

\section{Future}

\subsection{How take next step}

The fact that technology is becoming truly pervasive implies a shift from a single user or single system to large-scale heterogeneous systems involving multiple devices and people collaborating at different spatial and temporal scales. The technological factors that could contribute to such a promising change are well known:

Extend a variety of touch capabilities to everyday mobile devices. Currently, smartphones are equipped with GPS, cameras, microphones, acceleration sensors, gyroscopes, magnetometers, and light sensors. In addition, Bluetooth and Wi-Fi scanning can be used to determine the presence of people and infrastructure. Many manufacturers are also considering including environmental sensors, such as temperature or air pressure.

Ubiquity of mobile Internet connectivity. Most smartphone users today have a permanent Internet connection, so downloading data is possible almost anywhere and at any time, given the limitations associated with the volume of traffic.

A fusion of mobile computing and social media, which increasingly include automatically generated contextual information. Some examples include services like Foursquare, Facebook, and LinkedInthat makethe user's location visible on the Internet.

Thus, millions of complex, networked, mobile sensor systems are distributed all over the world. Methods for motivating users, collecting, evaluating, and using huge amounts of sensory data at the community level can be useful thanks to a constantly online user base. People are also increasingly willing to contribute data to support voluntary geographic information in order to increase the 
amount of data available to analyze urban dynamics while maintaining their privacy about the data they receive. In recent years, the perception of human activity through mobile devices has grown in various fields. This approach allows us to quantify a varietyof phenomena. To date, many methods of sensing and analyzing this activity in the geospatial environment have been proposed [2, 3]. The ability to perceive the state of various factors, such as specific particles, human flow, environmental conditions, etc., has contributed to mitigating the consequences of natural disasters, such as earthquakes, fires, epidemic control and prevention, roadcongestion, and urban planning [4].

\subsection{Clever city}

Urban computing for urban infrastructure planningis one of the most important areas in urban computing. It has mostly been applied either in relatively homogenous rural areas, where researchers have added sensors in specific locations, or in small, well-defined areas of development, such as smart homes or rooms. Urban areas are more complex and interesting spaces because they offer a lot of information about the dynamics of urban development. While urban environments tend to be much more dynamic in terms of what and who will participate in an app or system, urban spaces also provide us with many opportunities to explore new systems and applications that make life easier for people and those who serve the city. Emerging from this environment, urban computing gives researchers a new computational concept where every sensor, person, vehicle, building, and street in urban areas can be used as a computational component to serve people and the city.

Thus, in this paper we consider the concept of a software system for processing and analyzing big data from IoT devices, which will allow us to design and develop a software system for assessing the development of human settlements, which allows us to make a comprehensive assessment of energy consumption for the development of human settlements in terms of completeness and reliability of the initial information. The paper will consider models that can serve as a basis for developing a software packagea for processing and analyzing big data from IoT devices.

Serious work in the direction of the Internet of Things seems promising and interesting, which can be useful in many areas. Including in the energy sector.

Using the technology of the Internet of Things, it is possible to quickly and efficiently determine the problem areas of parts of the zones and draw up a competent map of the area, which will allow us to assess the scale of action to improve the living standards of people.

\section{References}

1. Torres-Ruiz M. J., Lytras M. D. Urban computing and smart cities applications for the knowledge society //International Journal of Knowledge Society Research (IJKSR). - 2016. - T. 7. - №. 1. - CP. 113-119.
2. Goodchild M. F. Citizens as sensors: the world of volunteered geography //GeoJournal. - 2007. - T. 69. - №. 4. - CP. 211-221.

3. Guo H., Goodchild M. F., Annoni A. Manual of digital Earth. - Springer Nature, 2020. - CP. 852.

4. Maeda H. et al. Generative adversarial network for road damage detection //Computer-Aided Civil and Infrastructure Engineering. - 2021. - T.36. - no. 1. pp. 47-60. 